EPiC Series in Health Sciences
Volume 1, 2017, Pages 139-143

\title{
Development of a Haptic System to Assess Wrist Control in Healthy and Injured Test Subjects
}

\author{
Xianghua Liu, BEng ${ }^{1}$, Stuart Bowyer, $\mathrm{PhD}^{2 *}$, and Joshua W Giles ${ }^{3 *}$ \\ ${ }^{1}$ Mechanical Engineering, Imperial College London, U.K. \\ ${ }^{2}$ Mechatronic in Medicine Lab, Imperial College London, U.K. \\ ${ }^{3}$ Wearable Technologies Lab, Imperial College London, U.K. \\ joshua.giles@imperial.ac.uk
}

\begin{abstract}
This paper presents the initial assessment of the feasibility of using a Sigma 7 teleoperation haptic device to replicate clinical wrist assessment tests and for the novel assessment of wrist proprioceptive control. Included in this paper is an assessment of the biomechanical suitability of the haptic device, as well as the control techniques that will be used to replicate clinical assessments. Finally, we conclude with proof of concept trials.
\end{abstract}

\section{Introduction}

Wrist injuries are common, with a recent study showing that 32-37\% of young athletes have suffered wrist pain (Kox, 2015). Current wrist assessment methods follow a general pattern of 'look, feel and move', and typically include Active (AROM) and Passive Range of Motion (PROM) assessments and resisted testing (Porretto, 2016; Arthritis Research, 2017). These tests are quick and simple but highly subjective and qualitative rather than quantitative (Lincoln, 1991; Rawes, 1996). Specifically, clinicians assess the 'end-feel' of a patient's wrist but this is highly subjective/qualitative, as well, joint angle measurements are commonly made visually but this has been shown to provide inconsistent results (McVeigh, 2016; Magee, 2008). All of this highlights the need to improve assessment methods with the aid of modern technology.

Proprioceptive impairments, which are commonly associated with nerve compression syndromes and ligament injuries, can severely affect the wrist's gross and fine motor function (Cappello, 2015; Lubahn, 1998) and reflexive control of balance, including passive and active motion sense (Goldscheider, 1898). Current clinical proprioceptive tests (e.g. Nottingham Sensory Assessment

\footnotetext{
${ }^{*}$ These two authors are considered co-senior authors.
} 
(Lincoln, 1998)) are coarse, indicating the need for a more objective, accurate and reliable method to sense force, motion and joint position (Cappello, 2015).

This study focuses on the feasibility of using existing haptic devices to transform qualitative wrist assessments (e.g. AROM, PROM, resisted and proprioceptive testing) to highly quantitative tasks with high data validity and reliability.

\section{Materials \& Methods}

Haptic device: A Sigma.7 (Force Dimension Inc.) haptic device with 7 degrees-of-freedom, which provides gravity compensation and force-and-torque feedback within a fully accessible $140 \mathrm{~mm}$ diameter sphere workspace, was assessed with respect to its feasibility for use as a wrist assessment tool (Figure 1). A rigid arm-rest was installed on a tripod such that the participant's forearm could be constrained with Velcro strips thus isolating wrist motion.
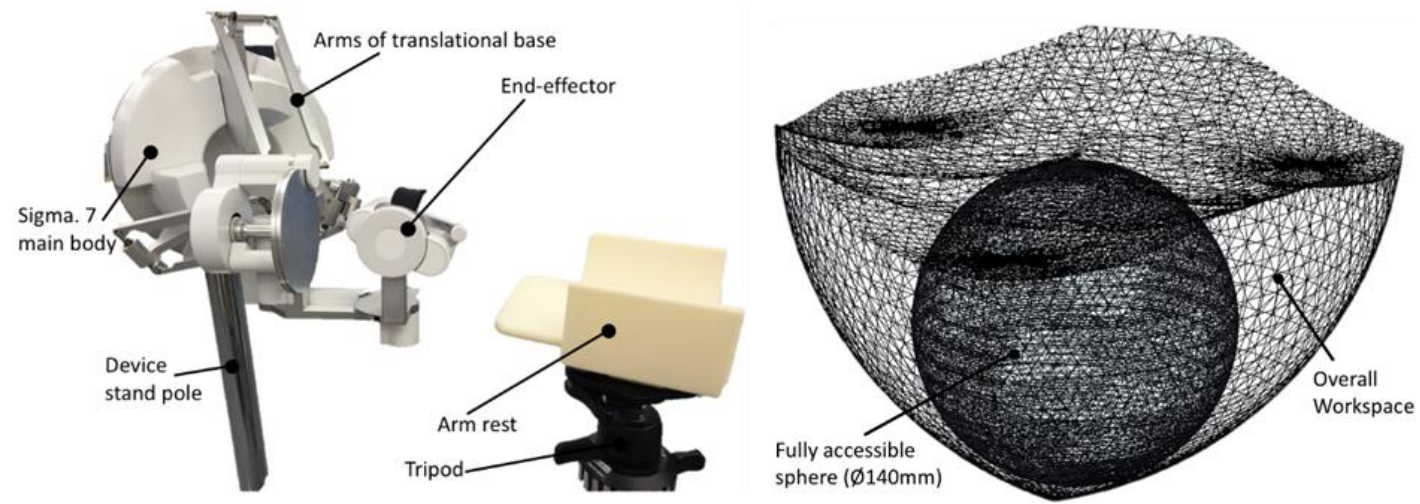

Figure 1. Left: The Sigma.7 haptic device and arm rest stand. Right: Workspace 3D Matlab model.

Work space and loading analysis: A Matlab 3D model (Figure 1b) of the workspace was built to verify that the clinical assessment motions (calculated from the range of wrist motion (Nordin, 2001) and average subject hand size (Dreyfuss, 1967) fit into the workspace. Additionally, given that there is a virtual centre of wrist rotation that lies at the centre of curvature of the distal radius bone (Taylor, 1955), the motion trajectories were assumed to be on a sphere surface centered at the wrist joint. Through collection of end effector position data during an initialization process, it is possible to determine the position of the patient's wrist center and thus apply physiologically meaningful motions and loads during wrist assessments. The haptic device can apply forces up to $20 \mathrm{~N}$, which equates to a maximum torque of $2.4 \mathrm{Nm}$ at the wrist, based upon the hand size of an average male (Dreyfuss, 1967). Previously, the literature has reported mean torques of $0.895 \mathrm{Nm}, 1.37 \mathrm{Nm}, 0.71 \mathrm{Nm}$ and $0.70 \mathrm{Nm}$ as being required to move a participant's wrist in passive flexion/extension/radial deviation (RD)/passive ulnar deviation (UD) to a full range of motion, respectively (Formica, 2012). Therefore, the Sigma.7 device provides a sufficient loading capacity to achieve passive wrist assessments.

Haptic control: For the PROM assessment, it is important that the applied torque matches the instantaneous stiffness of the patient's wrist to ensure smooth motion. This can be achieved by developing a controller to adjust the torque based upon the level of wrist passive stiffness (Formica, 2012) and the desired angular velocity of the joint. To guarantee safety, a function was developed to confirm that the applied force/torque and its derivative were below preset maxima. Additionally, this 
function prevents the application of motion/load if the patient releases the button on the end-effector or the examiner activates an 'emergency stop'.

Proof of concept tests: Two tests were carried out to assess the feasibility of the concept. Firstly, a participant was asked to hold the end-effector and perform flexion, extension, RD and UD motions, and the trajectories were plotted with respect to the device's 3D workspace model to investigate if the motions are fully contained within it. Secondly, the value of the device in conducting proprioceptive evaluations was tested by occluding the participant's vision and having them perform the Dart Throwers' motion (Anderson, 2016) which is a clinically relevant test that couples the wrist's rotational degrees of freedom. A number of loading conditions were simulated 1. no applied load, 2. constant perturbing force ( $2 \mathrm{~N}$ wrist extending force), 3. variable force disturbance ( $2 \mathrm{~N}$ magnitude sinusoidal flexion-extension force). During these trials, the hand trajectories were recorded and later compared to determine if the patient could sense and compensate for the perturbation.

\section{Results}

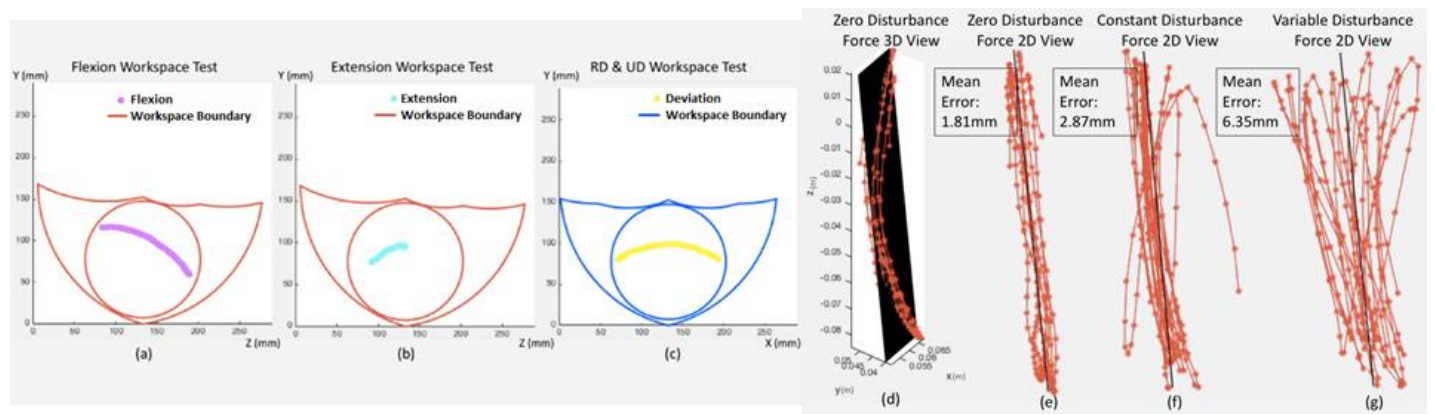

Figure 2. Proof of concept tests (Left: (a) Flexion Workspace Test, (b) Extension Workspace Test, (c) RD \& UD Workspace Test; Right: (d). 3D view of a zero disturbance trajectories on the motion plane, (e) Zero Disturbance proprioceptive feedback with mean error to the motion plane, (f) Constant Disturbance Proprioceptive feedback with mean error to the motion plane, (g) Variable Disturbance Proprioceptive feedback with mean error to the motion plane)

\section{Discussion}

We have introduced a haptic application for objectively, repeatably, and quantitatively assessing wrist function and control. Our initial proprioceptive motion trials as well as our workspace and load capacity verification demonstrate the feasibility and potential for the further development of a haptic application to aid clinicians in more effectively and accurately assessing wrist injuries which can compromise motion, function, and control. Specifically, we found that the Sigma.7 haptic device has a sufficiently large free motion workspace to permit the most important wrist assessment motions (e.g. flexion, extension, RD and UD). Additionally, our assessment of the device's loading capacity indicates that it should be capable of PROM and AROM tests as well as resisted motions with a pain initiation rather than maximum strength end point. With both of these points in mind, this device is capable of replicating the current systematic evaluation approach which integrates these three test types (Arthritis Research, 2017). 
Our initial proprioceptive evaluation verified the system's capability to produce three loading cases (zero, constant, and variable) that can, for the first time, provide an objective and quantitative means to assess a participant's ability to sense and react to external force through proprioception (Cappello, 2015). Although an optimal method for evaluating the patient's control ability under the three loading cases is still being identified, it is clear from the results in Figure 2 that using this haptic application can provide much great information than traditional qualitative techniques. In the future, the haptic application will be developed to carry out resisted and PROM assessments using the established wrist location identification algorithm and extend the proprioceptive evaluation to access the patient's ability to sense joint position.

\section{References}

Anderson Hoy G. Orthotic intervention incorporating the dart-thrower's motion as part of conservative management guidelines for treatment of scapholunate injury. Journal of Hand Therapy. 29(2):199-204. 2016.

Cappello L, Elangovan N, Contu S, Khosravani S, Konczak J, Masia L. Robot-Aided Assessment of Wrist Proprioception. Frontiers in Human Neuroscience. 9:1-2, 2015.

Clinical assessment of the musculoskeletal system. 1st ed. Chesterfield: Arthritis Research UK; 2017.

Dreyfuss H. The measure of man. 1st ed. New York, N.Y.: Whitney Library of Design; 1967.

Formica D, Charles S, Zollo L, Guglielmelli E, Hogan N, Krebs H. The passive stiffness of the wrist and forearm. Journal of Neurophysiology. 108(4):1158-1166, 2012.

Goldscheider A. Physiologie des Muskelsinnes. 1st ed. Leipzig: Barth; 1898.

Kox L, Kuijer P, Kerkhoffs G, Maas M, Frings-Dresen M. Prevalence, incidence and risk factors for overuse injuries of the wrist in young athletes: a systematic review. British Journal of Sports Medicine, 49(18):1189-1196, 2015.

Lincoln N, Crow J, Jackson J, Waters G, Adams S, Hodgson P. The unreliability of sensory assessments. Clinical Rehabilitation. 5(4):273-282, 1991.

Magee D. Orthopedic physical assessment. 1st ed. St. Louis, Mo.: Saunders Elsevier; P.35. 2008.

Lincoln, N., Jackson, J., and Adams, S. Reliability and revision of the Nottingham sensory assessment for stroke patients. Physiotherapy 84, 358-365, 1998.

Lubahn J, Cermak M. Uncommon Nerve Compression Syndromes of the Upper Extremity. Journal of the American Academy of Orthopaedic Surgeons. 6(6):378-386, 1998.

McVeigh K, Murray P, Heckman M, Rawal B, Peterson J. Accuracy and Validity of Goniometer and Visual Assessments of Angular Joint Positions of the Hand and Wrist. The Journal of Hand Surgery. 41(4): e21-e35, 2016. 
Nordin MFrankel V. Basic biomechanics of the musculoskeletal system. 1st ed. Philadelphia: Lippincott Williams \& Wilkins; 2001.

Porretto-Loehrke A, Schuh C, Szekeres M. Clinical manual assessment of the wrist. Journal of Hand Therapy. 29(2):123-135, 2016.

Rawes M, Richardson J, Dias J. A new technique for the assessment of wrist movement using a biaxial flexible electrogoniometer. The Journal of Hand Surgery: Journal of the British Society for Surgery of the Hand. 21(5):600-603, 1996.

Taylor C.L., Robert J. Schwarz. The Anatomy and Mechanics of the Human Hand. P22-35. 1955. 